\title{
NOVEDADES EN LA PARTICIPACIÓN PÚBLICA DE LAS ORGANIZACIONES VOLUNTARIAS DE DISCAPACIDAD. ÓRGANOS DE PARTICIPACIÓN
}

\section{PAULINO AZÚA}

Director de FEAPS (Confederación española de organizaciones en favor de las personas con discapacidad intelectual).

\section{INTRODUCCIÓN}

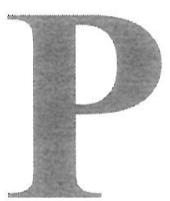

or lo general, general, las organizaciones voluntarias que actúan en el campo de la discapacidad han tenido tres objetivos primordiales: en primer lugar, la atención a las propias personas con discapacidad, así como la ayuda mutua entre ellos y sus familias. Por otra parte, han actuado con mayor o menor éxito en el ámbito de la información y de la comunicación, con el fin de modificar las actitudes sociales en torno a un problema que requiere profundos cambios por parte de la sociedad, y conseguir así que, quienes tengan una discapacidad, puedan disfrutar de unas condiciones de vida en situación de igualdad con los demás ciudadanos. Finalmente, las OO.VV. han tenido que actuar ante los poderes públicos-administraciones y grupos políticos-para propiciar la normativa y las decisiones que haga viables los objetivos que estas organizaciones persiguen.

Sin embargo, y a pesar de que este último aspecto constituye una preocupación permanente, a pesar de que en la trayectoria de estas organizaciones la acción política ha sido una constante en sus actividades, no se ha realizado de forma estructurada, a través de un sistema de participación más o menos estable, sino más bien ha sido fruto de relaciones individuales de los dirigentes de las propias organizaciones o de ocasiones puntuales debidas al impulso más que a la planificación.

Seguramente es en el sector de la discapacidad en el que se han producido con mayor antelación situaciones que auspiciaban la participación de las organizaciones voluntarias aunque, como no podía ser de otra manera, con perfiles sustancialmente distintos a los que hoy se producen.

El antecedente más remoto que yo conozco respecto de la participación de asociaciones en el campo de la discapacidad fue la creación 
de los Consejos Provinciales en el marco del INP (Instituto Nacional de Previsión) para el reconocimiento de las ayudas de 1.500 pts para "menores subnormales" otorgadas en aplicación del decreto que estableció en la Seguridad Social la asistencia a los menores subnormales en 1968. No puede decirse que estos consejos existieran con la finalidad de dar a las nacientes asociaciones de entonces "cancha política" puesto que aún estamos en 1968, pero sí es cierto que es una forma, bastante rudimentaria por cierto, de otorgar a estas asociaciones alguna presencia pública.

La regulación del antiguo SEREM del año 1971 dio lugar a la creación de una Comisión Asesora, con carácter consultivo, de la que formaba parte el Presidente de la Asociación Nacional de Inválidos Civiles. Posteriormente se creó el Consejo de Minusválidos que, si bien tenía un papel muy limitado, se abrió la participación de otras organizaciones del sector que al menos tenían acceso a una información que no estaba al alcance de todas las OO.VV.

Con verdadera dimensión política, la plataforma de participación más importante de que han dispuesto las OO.VV. dedicadas a la discapacidad ha sido el Real Patronato, creado en 1976 como de Educación Especial que, en su última reestructuración en 2000, pasa a denominarse Real Patronato sobre Discapacidad y en 2001 ha reestructurado sus órganos directivos. Durante estos veinticinco años, las organizaciones voluntarias han formado parte de la Junta de Gobierno de la institución y esa representación ha permitido hacer llegar, directamente, al ámbito de los Ministros integrantes de esa Junta de Gobierno las preocupaciones de los colectivos de personas con discapacidad. Sin embargo, y como consecuencia de la mencionada reestructuración, las organizaciones de personas con discapacidad han dejado de formar parte de la Junta de Gobierno y son convocadas a dicho órgano cuando se tratan temas que directamente les afectan.

\section{LA EDAD MODERNA DE LA PARTICIPACIÓN PÚBLICA}

En el ámbito general de la acción social, el precedente más próximo hay que buscarlo en los Consejos de Bienestar Social, nacidos al amparo de las respectivas leyes autonómicas de Servicios Sociales. La primera de ellas es la del País Vasco (6/1982, de 20 de mayo) y marca la tónica de lo que se regulará en las demás, creando un Consejo de ámbito autonómico, con carácter consultivo y en el que existe representación de las OO.VV. que actúan en el ámbito de la ley y, entre ellas, las organizaciones dedicadas a las personas con discapacidad. 
La creación de estos Consejos tiene la virtualidad de acostumbrar a las OO.VV, a los sectores profesionales y al sector público a compartir y a debatir orientaciones y puntos de vista, en definitiva, a encontrarse en un terreno en el que, muchas veces, hasta ese momento sólo se había mantenido en términos estrictamente dialécticos. Sin embargo, la opinión de las asociaciones que han formado parte de estos consejos es que, con carácter general, no han resultado particularmente operativos.

Desde mi punto de vista, la participación cobra verdadera eficacia cuando es el sector el que se organiza a sí mismo, sin especiales interferencias externas, como consecuencia de una toma de conciencia sobre la fuerza política que cobran las organizaciones cuando actúan de manera coordinada y conjunta. Este me parece un elemento nuevo en el sector de la discapacidad que quiero destacar: las OOVV se han dado cuenta que el acceso a los poderes públicos pasa, cada vez de forma más intensa, por la actuación conjunta, lo que lleva a estas organizaciones a una cesión parcial de su soberanía. Naturalmente, no siempre esta cesión resulta bien acogida por parte de algunos dirigentes, anclados en posturas en las que el contacto con los centros de decisión política, se convierte en un fín en sí mismo, más allá o con independencia de si se alcanzan los objetivos o no.

Por otra parte, no creo que ofrezca mayor discusión un hecho que me parece evidente: la capacidad política de las organizaciones, si se pretende desarrollar de forma aislada, pierde fuerza. Se podrán abordar problemas puntuales, pero no cuestiones políticas de mayor profundidad. Todo ello, sin olvidar que una parte muy importante de las medidas políticas están orientadas a la generalidad del mundo de las personas con discapacidad, por lo que la acción de las OO.VV. debe tener ese carácter general. Podríamos hablar de la "globalización" del sector en lo referente a las medidas que precisa y, en consecuencia, a la acción política de las organizaciones que lo integran.

Quiero citar dos experiencias de participación de las OOVV basadas, precisamente, en esta conjunción de esfuerzos a la que me vengo refíriendo, una referida específicamente al ámbito de la discapacidad y otra, más amplia, al de las Organizaciones no Gubernamentales que actúan en el campo de la acción social, la primera porque está constituida únicamente por entidades que actúan en este campo y la segunda, más amplia, porque de la misma forman parte organizaciones de personas con discapacidad. 


\subsection{El Comité Español de Representantes de Minusválidos}

En el primer caso, el exponente al que me voy a referir es el Comité Estatal de Representantes de Minusválidos - CERMI, compuesto únicamente por organizaciones que actúan en el sector de la discapacidad y la Plataforma Estatal de ONG de Acción Social de la que varias organizaciones de minusválidos forman parte.

En su aún corta historia, el CERMI ha pasado por tres etapas:

1. Como plataforma de hecho, compuesta por organizaciones que habían venido colaborando con el Real Patronato y que a finales de los 80 decidieron reunirse periódicamente para cambiar puntos de vista y hacer algunos planteamientos comunes ante el Gobierno. Ya desde esos primeros momentos el CERMI adoptó una denominación que ha sobrevivido a los cambios. Este rudimento de plataforma de actuación no tuvo una excesiva importancia externa -todo hay que decirlo- pero sí interna en la medida en que constituyó la base de entendimiento necesaria para crear jurídicamente el CERMI y comenzar un trabajo de representación eficaz.

2. La incorporación de España a la Comunidad Europea hizo necesario que en España, al igual que venía sucediendo en otros países, se creara un comité de coordinación de las entidades más importantes del sector de la discapacidad. La solución se antojaba fácil puesto que la plataforma ya estaba formada y sólo parecía necesario redactar los estatutos y completar la incorporación de algunas entidades, hasta entonces ausentes. Ahí comenzaron los problemas ante la negativa de alguna organización "de" minusválidos a que formara parte del CERMI otra organización "para" minusválidos. La solución fue paradójica. Con la inclusión de ambas organizaciones discrepantes y, puesto que era necesario crear la plataforma para poder organizar actuaciones en el marco de la Comunidad Europea, se optó por redactar los estatutos que fijaban el ámbito de actuación del CERMI fuera de España, en el marco de la CE, pero impedian su funcionamiento de puertas adentro. Así se escribe la historia.

3. Naturalmente, una situación así no podía mantenerse mucho tiempo, por lo que, al final, se impusieron la razón y el sentido común y se "recreó" el CERMI con seis miembros fundadores' y con unos estatutos a los que cabría calificar como "normales" en vista de lo anómalos que habían sido los anteriores. A partir de esta reforma el CERMI agrupa a más de 2.000 asociaciones y entidades que re- 
presentan en su conjunto a los tres millones y medio de personas con discapacidad que, según las estadísticas hay en España. ${ }^{2}$

A partir de ese momento, el CERMI se ha ido configurando, sobre todo, como un lobby, como un instrumento para la acción política y, en consecuencia, sus referentes básicos son tanto los ministerios del gobierno de la nación con responsabilidades en el área de la discapacidad como los diferentes grupos parlamentarios y partidos políticos. Esta plataforma ha optado claramente por esta línea de trabajo, soslayando una opción que podría parecer tentadora pero que encierra en sí misma un grave peligro: actuar como entidad de gestión y propiciar la elaboración y gestión de programas que beneficien a sus organizaciones miembros.

En este último periodo, el CERMI ha trabajado en una doble dirección: su cohesión interna y la representación de los intereses generales de las personas con discapacidad. Como organización se ha visto reforzada con la incorporación de otras entidades que, sin tener el carácter de fundadores, se han incorporado como miembros ordinarios del Comité. Por otra parte, se ha extendido el modelo a la práctica totalidad de las Comunidades Autónomas, de forma que existen CERMIs autonómicos compuestos de forma análoga al estatal que están comenzando a desarrollar una importante labor en sus respectivos ámbitos de actuación.

Por lo que se refiere a los logros alcanzados en el quehacer político, el CERMI puede incorporar a su haber el primer acuerdo con el Gobierno en materia de empleo, los beneficios fiscales obtenidos para las personas con discapacidad en las reformas desarrolladas en los últimos años, así como puntuales avances recogidos en las leyes de acompañamiento de los Presupuestos Generales. Como novedad hay que señalar el segundo acuerdo en materia de empleo firmado entre el CERMI y el Ministerio de Trabajo y Asuntos Sociales el pasado 3 de diciembre. Además, la nueva reforma del IRPF que entrará en vigor en este año 2003 también recoge beneficios para las personas con discapacidad por impulso del CERMI.

El CERMI se ha convertido en un referente obligado en lo que tiene de esfuerzo de coordinación y de trabajo conjunto así como de instrumento de presión ante la Administración y los partidos políticos. Sus actuaciones en estos ámbitos nacen de una acreditada voluntad de cooperación, aunque esta cooperación deba ser crítica en determinados momentos. 


\subsection{La Plataforma de $O N G$ de Acción Social}

La segunda experiencia de participación que me parece reseñable, en un marco más amplio de actuación, la constituye la Plataforma de ONGs de Acción Social. Esta Plataforma se origina a partir del proceso de diálogo con la Presidencia del Gobierno iniciado en 1998. El 21 de mayo de ese año, el Presidente del Gobierno convoca a 16 ONGs del ámbito social a una reunión en la que se ponen de relieve las necesidades del sector. Se inicia así un proceso de encuentros en el que por parte de las organizaciones se pide un marco legislativo favorable, con recursos, capacidad de gestión e instrumentos de participación, entendiendo que el proceso de reforma es estructural y debe hacerse con el máximo consenso social, político e institucional. Apenas un año después, el 12 de enero de 1999 se produce un nuevo encuentro con el Presidente del Gobierno en el que, desde las ONGs se plantean seis propuestas para la reforma del tercer sector: elaboración de un Libro Blanco del Tercer Sector, impulsar un Plan Estratégico de Acción Social, constitución del Consejo estatal de ONGs de Acción Social, medidas de Apoyo al Tercer Sector a través de la reforma de la asignación del 0,52\% del IRPF, reforma de la Ley de Fundaciones de 1994 y elaboración de una nueva Ley de Asociaciones. El 18 de mayo de 2000 se constituye la Plataforma de ONGs de Acción Social ${ }^{3}$ en la que están integradas organizaciones específicas y otras de carácter general, con diferentes grados de implantación y de posibilidades de actuación pero todas con el denominador común de su actuación en el ámbito del sector social y de poner en práctica programas financiados con cargo al $0,52 \%$ del IRPF.

\section{PLATAFORMA DE ONG DE ACCIÓN SOCIAL}

\begin{tabular}{|lr|}
\hline Personas asociadas & 3.283 .000 \\
Personas voluntarias & 319.000 \\
Personal remunerado & 90.650 \\
Usuarios & 10.630 .000 \\
\hline
\end{tabular}

Las entidades que la integran son: AECC (Asociación Española contra el Cáncer), ATIME (Asociación de Trabajadores Inmigrantes Marroquíes en España), Cáritas Española, COCEDER (Confederación de Centros de Desarrollo Rural), COCEMFE (Confederación Coordinadora Estatal del Minusválidos Físicos de España), FEAPS (Confederación Española de Organizaciones en Favor de las Personas con Discapacidad Intelectual), CNSE (Confederación Nacional de Sordos de España), Cruz Roja Española, FMP (Federación de Mujeres Progresistas), FEMUR (Federación Nacional de la Mujer Rurl), Fundación ESPLAI (Acción Social. Educación. Tiempo Libre), Fundación Juan Ciudad (Orden Hospitalaria de San Juan de Dios), ONCE (Organización Nacional de Ciegos Españoles), UDP (Unión Democrática de Pensionistas y Jubilados de España), UNAD (Unión Española de Asociaciones y Entidades de Atención al Drogodependiente) y UR (Unión Romaní). 
La Plataforma ha negociado con el Gobierno de España un nuevo marco económico que, apoyado en el IRPF, asegura un mínimo de financiación que permite que los programas realizados puedan tener razonables garantías de continuidad. Este acuerdo de carácter trienal finaliza en la presente campaña del impuesto y se está negociando un nuevo acuerdo que permita avanzar por este camino durante los próximos años. Asimismo, la Plataforma ha propiciado la elaboración del Libro Blanco sobre el Tercer Sector que verá la luz en los primeros meses de este año 2003, ha intervenido en la reforma de la Ley de Fundaciones, me atrevo a decir que de manera importante en la nueva Ley de Asociaciones y ha conseguido que el Gobierno impulse la creación del Consejo Estatal de ONGs de Acción Social.

El balance de resultados es importante, aunque como es natural no se hayan alcanzado en su plenitud los objetivos deseados, lo que entra de lleno en la normalidad del quehacer de todas las organizaciones. La Plataforma tiene ante sí el reto de dar respuesta a algunas organizaciones que están llamando a su puerta para integrarse en la misma. El problema para ello es que los miembros actuales de la Plataforma han conseguido un sistema de entendimiento y de trabajo en común que podría quebrarse con nuevas incorporaciones si éstas no son capaces de compartir el mismo talante de pacto, de cesión de protagonismo en beneficio de un objetivo común.

\section{LO PRIVADO DA LUGAR A LO PÚBLICO}

La coordinación de las organizaciones privadas, la creación de plataformas para la acción conjunta como las que acabo de describir generan procesos similares en el ámbito de lo público y para los mismos sectores a que he hecho mención. Quiero destacar dos casos:

\subsection{Consejo Estatal de la Discapacidad.}

Este Consejo arranca, curiosamente, del "Acuerdo sobre medidas urgentes para la promoción del Empleo de las Personas con Discapacidad" suscrito entre el Ministro de Trabajo y las organizaciones fundadoras del CERMI el 15 de octubre de 1997. La creación de este Consejo fue propuesta por el Ministerio de Trabajo y aceptada por las organizaciones a pesar de que no constituía ninguna demanda urgente por parte del sector. ${ }^{4}$ Se crea mediante Orden de 17 de junio de 1999 (casi dos años después de haberse acordado) y tiene como fin

4 Por otra parte, pactar la creación de un órgano consultivo de carácter general en un acuerdo sobre empleo no parece excesivamente coherente. 
fundamental el institucionalizar la colaboración del movimiento asociativo de personas con discapacidad y de la Administración General del Estado para coordinar y definir una política coherente de atención integral.

El Consejo está compuesto por veintiseis vocales de los que trece lo son en representación de la Administración Central del Estado y trece en representación del movimiento asociativo. De éstos, doce son integrantes del CERMI - lo que da una idea de la cohesión asociativa en torno a esta plataforma-y uno está en representación de la asociación ANDE.

Aunque la norma de constitución determina que el Pleno del Consejo celebrará al menos tres sesiones ordinarias al año y la Comisión Permanente se reunirá cada tres meses en sesión ordinaria, el hecho es que hasta enero de 2003, sólo se ha reunido el Pleno una vez, el 16 de febrero de 2001 para el acto de constitución, y la Permanente ninguna. La explicación a este retraso se me antoja doble: por una parte, por los sucesos acontecidos en torno a la asociación mencionada, que ${ }^{5}$ han provocado que los integrantes del Consejo en representación del CERMI hayan manifestado su voluntad de no reunirse con el representante de una asociación que está encausado y, por otra parte, la representación pública tampoco ha manifestado una excesiva preocupación en resolver jurídicamente esta situación.

A pesar de ello, el CERMI ha seguido actuando en su papel de representación de la globalidad del movimiento asociativo, alcanzando diferentes acuerdos con diversos órganos de la Administración Central y dialogando con los partidos políticos, lo que avala mi comentario anterior en el sentido de que, más allá de los órganos consultivos y de participación que se constituyan en el ámbito de lo público, que en sí mismo son importantes, lo verdaderamente necesario es que las organizaciones y asociaciones estén coordinadas entre sí y actúen de manera conjunta en su función de "lobby".

\subsection{Consejo Estatal de ONG de Acción Social}

Este Consejo se crea en virtud del Real Decreto de 17 de diciembre con carácter de órgano de encuentro, diálogo, participación y asesoramiento, y con funciones de asesoramiento y propuesta de medidas de

Según noticias de prensa, el presidente de la Asociación ANDE ha sido denunciado por la Fiscalía de Madrid por malversación de fondos públicos, apropiación indebida, estafa y falsedad, por importe de 750 millones de pesetas por no haber acreditado ni entregado los libros de contabilidad entre los años 1997 y 2000. Esta denuncia se ha producido en diciembre de 2002 y de la misma se han iniciado las diligencias previas en el juzgado de instrucción $n^{0} 39$ de Madrid. 
política social en favor de los grupos vulnerables de la sociedad. Está compuesto por treinta y dos miembros de los que veintidos ${ }^{6}$ lo son en representación de diferentes organizaciones no gubernamentales y diez de diferentes órganos de la Administración del Estado.

Desde su constitución, el Consejo se ha reunido en varias ocasiones, algunas vinculadas monográficamente a las convocatorias de subvenciones con cargo al $0,5 \%$ de la asignación tributaria del IRPF y otras a un plan de trabajo que se ha elaborado en el marco del Consejo.

Para la formulación y desarrollo del mismo se han creado cinco grupos de trabajo sobre las siguientes materias: Financiación, Empleo, Calidad, Inclusión Social y Familia. Todos ellos han elaborado sus propuestas que están pendientes de debatir y, en su caso, aprobar en una próxima reunión del Consejo prevista para comienzos de 2003.

Como puede comprobarse, este órgano de participación se está mostrando más activo que el de Personas con Discapacidad. Ello se debe, en parte, a que algunos de los grupos han recogido el testigo de algunas materias que ya venían siendo tratadas por la Plataforma de ONG y, también en parte, a que algunos temas habían sido objeto de estudio por parte de organizaciones del sector.

Conviene reseñar que las organizaciones integrantes del Consejo han puesto de relieve el hecho de que el sistema de financiación es manifiestamente mejorable. En efecto, supeditar la política social que emana del Ministerio de Trabajo y Asuntos Sociales - no la que desarrollan las Comunidades Autónomas en el marco de sus competencias- a la manifestación de voluntad de los contribuyentes a la hora de cumplir sus deberes fiscales podría llevar al absurdo de que si éstos no manifiestan su interés en hacerlo como hasta ahora, a través del IRPF, la política social desarrollada por el MTAS a través de las ONG carecería de financiación.

Este comentario en torno a la financiación pone de relieve que las $\mathrm{ONG}$, en tanto que integrantes del Consejo Estatal, las organizaciones

6 Las OOVV que forman parte del Consejo Estatal son las siguientes: Cruz Roja Española, Cáritas Española, Plataforma de ONG de Acción Social, Confederación de Centros de Desarrollo Rural, Plataforma de Organizaciones de Infancia, Unión de Asociaciones Familiares, Federación de Mujeres Progresistas, Federación Nacional de la Mujer Rural, Federación de Scouts de España ASDE, Organización Juvenil Española, Unión Democrática de Pensionistas y Jubilados, Federación de Asociaciones de Mayores de Canarias, Confederación Coordinadora de Minusválidos Físicos de España, Confederación Española de Organizaciones en favor de las Personas con Discapacidad Intelectual, Comité Español de Representantes de Minusválidos, Unión Española de Asociaciones y Entidades de Atención al Drogodependiente, Asociación UNIVER-SIDA, Asociación Secretariado Nacional Gitano, Unión Romaní, Plataforma para la Promoción del Voluntariado en España, Comisión Española de Ayuda al Refugiado, Asociación de Trabajadores Inmigrantes Marroquies en España. 
voluntarias no se limitan a una función consultora o de asesoramiento sino que ejerce con cierta firmeza su papel de propuesta de medidas que tiendan a mejorar el sector. El tiempo dirá si otras acciones en las que se está trabajando en los diferentes grupos, como es el acercar las ONG a planteamientos de calidad en sus actuaciones o el empleo para los colectivos desfavorecidos son asumidas por los responsables de la Administración y resultan realmente eficaces.

\section{ALGUNAS CONSIDERACIONES FINALES}

La situación que he intentado describir da pie, a mi juicio, para algunos comentarios finales que pueden tener algún interés.

En primer lugar quiero destacar el hecho de que estas plataformas implican una importante cesión de soberanía por parte de las organizaciones que las integran que, en el terreno de la representación, se subsumen en planteamientos generales con los que, a cambio de perder protagonismo, llegan con mayor facilidad a instancias que de otra forma resultan menos accesibles. Ello no quiere decir que estas organizaciones no lleven adelante sus propios planteamientos de forma individual, pero lo deseable es que esto se produzca cuando se trata de aspectos que únicamente afectan a una organización en concreto y que, por tanto, tienen menos encaje en enfoque general.

Para que esta función representativa y de participación resulte eficaz, es necesario que se ciña al ámbito de lo político lo que implica renunciar a la gestión. Si estas plataformas, sean en el ámbito estatal o en el autonómico, se ven tentadas por proyectos que impliquen gestión de subvenciones estarán condenadas al fracaso. El difícil equilibrio alcanzado en el terreno de las estrategias se verá complicado por un debate sobre recursos -siempre escaso- que sin duda hará de cortocircuito en una tarea hasta ahora desempeñada de forma satisfactoria.

Desde el punto de vista de la estrategia de las organizaciones, disponer de una plataforma conjunta protege a éstas, en algunas ocasiones, de las respuestas de una Administración poco proclive a asumir la crítica y, en consecuencia, a adoptar medidas contra dicha organización. Se ha dicho muchas veces que una asociación cuya fuente de ingresos proviene fundamentalmente de las subvenciones públicas se puede ver en situación desamparada a la hora de contradecir y, en el ejercicio de su función, criticar el quehacer público. Pues bien, un planteamiento conjunto diluye en un cuerpo común la responsabilidad de la crítica, que puede ser hecha por el común de organizaciones incorporadas a una plataforma, en la que asumen colectivamente esa responsabilidad. 
No sólo ante los poderes públicos. También ante la sociedad una voz única constituye un potente altavoz para hacer llegar determinados mensajes. Desde esta perspectiva, los destinatarios de esos mensajes perciben un cambio fundamental en el que las organizaciones voluntarias pierden una parte de su carácter autárquico, de reinos de taifas y dan respuesta a un sentir social que viene pidiendo, desde hace mucho tiempo que se produzca una voz común que denuncie la realidad y que, con ese mismo carácter, oriente sus respuestas a los problemas sociales.

Naturalmente, a esta situación no se llega ni se mantiene sin esfuerzo. Actuar desde la colaboración requiere nuevas energías por parte de los responsables de las organizaciones que se ven enfrentados a la gestión del entorno, camino todavía muy largo por recorrer. Por otra parte, este tipo de planteamientos facilita enormemente que las organizaciones, aisladamente, no se empeñen en descubrir la rueda, que copien estrategias y planteamientos unas de otras y que, en definitiva, sean solidarias entre sí. En todo caso, no me parece que la senda emprendida pueda desandarse y, aunque aún hay muchas dificultades por salvar, la experiencia está demostrando que ésta es la palanca más importante para la acción pública de las organizaciones voluntarias. 